\title{
Carbon monoxide: not gone, not to be forgotten
}

\author{
John A Henry
}

Carbon monoxide is a remarkable poison. Because of the non-specific manner in which it may present, and the consequent multiplicity of possible diagnoses, it has been described as "the silent killer". This issue of the journal contains three publications on the topic, and if that were not enough, the Chief Medical Officer has recently written to all the general practitioners in Britain warning them of the problems associated with carbon monoxide poisoning. ${ }^{12}$ Another useful reference document has also appeared from the Health and Safety Executive outlining the diagnosis and management of carbon monoxide toxicity with an emphasis on the industrial aspects. ${ }^{3}$ It is necessary for all those working in accident and emergency departments to be aware of the diagnosis, the management, the pitfalls, and the science of carbon monoxide poisoning.

Some may ask why there is such concern about carbon monoxide. The reason is that, apart from being the commonest cause of fatal poisoning in Britain and many other countries, it is now well recognised as a cause of neurotoxicity. There may be clearly evident damage, such as parkinsonian syndromes, cortical blindness, and dementia, or there may be more subtle problems, such as apathy, fatigue, irritability, minor memory disturbances, difficulty with decisions, and personality change. ${ }^{45}$ It is not necessary for the patient to have suffered severe or life threatening poisoning for these effects to occur. Carbon monoxide is also unusual in that delayed neurotoxicity may occur days or even weeks after apparent recovery from the episode of poisoning. ${ }^{6}$

The most immediate problem is that of diagnosis. The clinical assessment of the patient may reveal very few clues. Headache, dizziness, fatigue, and nausea are common symptoms. Tachycardia, tachypnoea, postural hypotension, and signs of central nervous system depression occur in more severe cases. One should always suspect carbon monoxide when people present with non-specific symptoms, particularly in cold weather; unfortunately, this is precisely the time when viral and bacterial infections are more common. When more than one person has suffered typical symptoms, or if a pet animal has also become ill or died, the clinician should actively consider the diagnosis. ${ }^{7}$ If everyone in the premises has suffered from headache and

vomiting, then carbon monoxide poisoning is by far the most likely diagnosis. The carboxyhaemoglobin ( $\mathrm{COHb}$ ) concentration is the most important diagnostic test, and blood should be taken as early as possible after the patient presents, as the blood $\mathrm{COHb}$ declines rapidly, especially if the patient is being given oxygen by facemask. The paper by Turner, Esaw, and Clark (p96), ${ }^{8}$ which investigates the importance of metabolic acidosis, helps to confirm that acidosis is a significant feature of carbon monoxide poisoning and also that initial acidosis is a good predictor of severity and of treatment requirements. It is clear that the initial assessment of the patient should include a full history and clinical examination, a $\mathrm{COHb}$ concentration, and also an arterial $\mathrm{pH}$ estimation. However, this publication also shows us that better markers of intracellular hypoxia are needed. What is currently being measured reflects whole body hypoxia, but it is mainly cerebral hypoxia that is responsible for the acute presentation and long term sequelae.

The update by Turner, Hamilton-Farrell, and Clark ( $p 92)$ is a useful review of a range of new concepts in carbon monoxide poisoning. ${ }^{9}$ Ideas about the mechanism of toxicity have developed markedly. The original simplistic picture put forward by Haldane is no longer accepted. He believed that the toxicity of carbon monoxide was secondary to its high affinity for haemoglobin, and that the resulting decrease in the oxygen carrying capacity of the blood caused end organ hypoxia. Other mechanisms are much stronger candidates for causing symptomatology and toxicity. These include combination of carbon monoxide with cytochromes and with myoglobin, inhibition of mitochondrial metabolism, competition with nitrous oxide, promotion of free radical damage and inflammatory mechanisms; their precise contributions remain to be elucidated. However, these mechanisms are not easy to detect or identify in clinical practice, and because of its ease of measurement, blood $\mathrm{COHb}$ remains the most important diagnostic test despite its poor predictive value. The authors have posed questions about the roles of glucose and ethanol, but the relationship of these two to acute and chronic human poisoning is not clear.

Therapeutic interventions are limited to supportive care and normobaric or hyperbaric 
oxygen. Anecdotal reports of alternative approaches have not been followed up. ${ }^{10}$ There is little evidence from clinical studies that normobaric oxygen is effective, and even the evidence concerning hyperbaric oxygen is difficult to interpret despite animal studies and cellular research which provide strong evidence in its favour. One important factor is that it reduces free radical concentrations, and this means that the benefit from hyperbaric oxygen may be far reaching. It provides an explanation why hyperbaric oxygen prevents the delayed manifestations of carbon monoxide poisoning.

The presentation by Hamilton-Farrell of the British Hyperbaric Association's carbon monoxide database (p98) provides an impressive panorama of the use of hyperbaric oxygen in Britain. ${ }^{1}$ The indications for referral are clearly set out, and the reasons for delay to referral and delay to arrival in a hyperbaric facility are listed. Many of these can be reduced by making a critical examination at a local level and addressing the deficiencies identified. The rising number of referrals may be a result of increased awareness, since the number of cases of fatal poisoning is falling. It has been suggested that this is due to the introduction of catalytic converters in motor vehicles. $^{12}$

Those working in any branch of emergency medicine would do well to study the articles presented in this issue of the journal. One certainly wants to avoid the error of telling a patient with undiagnosed carbon monoxide poisoning to "go home and stay warm", with the result that they suffer even worse poisoning.

1 Chief Medical Officer and Chief Nursing Officer. Carbon monoxide: the forgotten killer. London: Department of monoxide: the forgotten
Health, September 1998.

2 Chief Medical Officer. CO poisoning: pulse oximetry. CMO's update 20. London: Department of Health, November 1998: 3.

3 Carbon monoxide: health hazards and precautionary measures. Guidance note EH43. 2nd Ed. London: Health and Safety Executive, 1998

4 Smith JS, Brandon S. Morbidity from acute carbon monoxide poisoning at three year follow up. BMF 1973;i:318-21.

5 Chale SN. Carbon monoxide poisoning. In: Vicciello P, ed. Handbook of medical toxicology. Boston: Little, Brown 1993: Handbook

6 Choi IS, Kim SK, Lee SS, et al. Evaluation of outcome of delayed neurologic sequelae after carbon monoxide poisoning by technetium $99 \mathrm{~m}$ hexamethylpropylene amine oxime brain singe photon emission computed tomography. Eur Neurol 1995;35:137-42.

7 Kirkpatrick JN. Occult carbon monoxide poisoning. West 7 Med 1987;146:52-6.

8 Turner M, Esaw M, Clark RJ. Carbon monoxide poisoning treated with hyperbaric oxygen: metabolic acidosis as a predictor of treatment requirements. F Accid Emerg Med predictor of treat

9 Turner M, Hamilton-Farrell MR, Clark RJ. Carbon monoxide poisoning: an update. $\mathcal{F}$ Accid Emerg Med 1999;16: ide poisc

10 Howard RJ, Lake DR, Pall H, et al. Allopurinol/Nacetylcysteine for carbon monoxide poisoning. Lancet 1987;ii: $628-9$.

11 Hamilton-Farrell MR. British Hyperbaric Association carbon dioxide database, 1993-96. F Accid Emerg Med 1999;16:98-103.

12 Kendell RE. Catalytic converters and prevention of suicide. Lancet 1998;352:1525.

\title{
Carbon monoxide poisoning: an update
}

\author{
Mark Turner, Martin R Hamilton-Farrell, Robin J Clark
}

Carbon monoxide is the major cause of death from poisoning in the UK. In 1996 there were 877 deaths from carbon monoxide poisoning (CMP) in England and Wales; the majority were suicides due to car exhaust fumes. ${ }^{1}$

\section{Department of Cardiology, Derriford Hospital, Plymouth, Devon PL6 8HD M Turner \\ Whipps Cross Hospital, London M R Hamilton-Farrell \\ Royal Naval Hospital Haslar, Gosport R J Clark \\ Correspondence to: Dr Turner, Specialist Registrar in Cardiology. deliberate and half accidental. ${ }^{5}$ This contrasts with the mortality figures, with $85 \%$ of CMP deaths being due to deliberate poisoning.
Death may be due to cardiac toxicity, neurotoxicity, systemic acidosis, or respiratory arrest.

\section{Pathophysiology}

The commonly held belief that CMP is mainly due to binding of haemoglobin was shown to be false over 20 years ago. ${ }^{6}$ Goldbaum et al demonstrated that exchange transfusion, using a red cell concentrate with a carboxyhaemoglobin $(\mathrm{COHb})$ concentration of $80 \%$, led to indefinite survival in one group of dogs, despite equilibrium $\mathrm{COHb}$ concentrations of $60 \%$. The potential to endure a diminished oxygen carrying capacity was demonstrated when other dogs were venesected isovolaemically, reducing mean packed cell volume by $68 \%$, and survived indefinitely. In contrast, dogs in a third group inhaled $13 \%$ carbon monoxide for 15 minutes, producing a mean $\mathrm{COHb}$ of $65 \%$, and all died. Goldbaum et al deduced that the slow rate of carbon monoxide binding by haemoglobin led to blood entering the systemic capillary beds with a

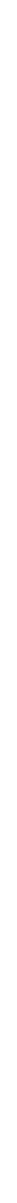

